\title{
Herschel and ALMA measurements of dust and molecules in Supernova 1987A
}

\section{Margaret Meixner $^{\star a b}$, Remy Indebetouw ${ }^{c d}$, Mikako Matsuura ${ }^{e}$, Julia Kamenetzky ${ }^{f}$, Richard McCray $^{f}$, Eli Dwek ${ }^{g}$ and the SN1987A Team ${ }^{\dagger}$}

${ }^{a}$ Space Telescope Science Institute, 3700 San Martin Dr., Baltimore, MD 21218, USA

${ }^{b}$ Johns Hopkins Unviersity, Dept. of Physics and Astronomy

${ }^{c}$ Department of Astronomy, University of Virginia, PO Box 400325, Charlottesville, VA, 22904, USA

${ }^{d}$ National Radio Astronomy Observatory, 520 Edgemont Rd, Charlottesville, VA, 22903, USA

${ }^{e}$ Dept. of Physics and Astronomy, University College London, Gower Street, London WC1E 6BT, $U K$

${ }^{f}$ Dept. of Astrophysical and Planetary Sciences, University of Colorado at Boulder, UCB 391, Boulder, CO 80309, USA

${ }^{g}$ NASA/GSFC, Code 665, Observational Cosmology Lab, Greenbelt, MD 20771-0001, USA

E-mail: meixner@stsci.edu, remy@virginia.edu,

mikako.matsuura@ucl.ac.uk, julia.kamenetzky@colorado.edu

jrichard.mccray@colorado.edu,eli.wek@nasa.gov

Dust production by supernovae is important in the dust life cycle of a galaxy. The explosion of SN 1987A was the nearest SN detected in the last 400 years, allowing us detailed studies of contemporary evolution of a supernova for the first time. In 2011, Matsuura et al. reported 0.40.7 M $\odot$ of dust in SN 1987A based on Herschel HERITAGE survey data, which is surprisingly large compared to prior measurements of supernovae. In this paper, we present our follow-up studies of this important discovery about SN 1987A using the Herschel Space Observatory and the Atacama Large Millimeter Array (ALMA). We highlight two important results, the detection of cold molecular gas and dust in the ejected material of SN 1987A. Our results suggest that SNe are significant producers of dust and molecules, as well as heavy elements, driving chemical evolution of galaxies.

The Life Cycle of Dust in the Universe: Observations, Theory, and Laboratory Experiments - LCDU 2013, 18-22 November 2013

Taipei, Taiwan

\footnotetext{
*Speaker.

†The SN1987A Team also includes: G. Zanardo, L. Staveley-Smith \& T. Potter(ICRAR); M. Barlow, J. Yates \& B. M. Swinyard (UCL); B. Arbutina, D. Urosevic \& V. Zekovic (U Belgrade); A. Bolatto (UMD); C. Vlahakis \& R. Wesson (ESO); R. Chevalier (UVa); C. Fransson (Stockholm Univ); D. Burrows (Penn State); M. Baes, J. Verstappen, I. De Looze, G. Gentile \& J. Fritz (Univ. of Gent); P. Bouchet, K. Okumura, \& M. Sauvage (CEA/Saclay); K.A. Misslet (UAz); H.L. Gomez (Cardiff Univ.); T. Lim (RAL); M.A.T. Groenewegen, G. Van de Steene \& P.A.M. van Hoof ( ROB); P. Royer (Inst. voor Sterrenkunde); G. Sonneborn (NASA/Goddard); G. Clayton \& E. Montiel (LSU); B. Gaensler (Univ. of Sydney); R. Kirshner \& A. Soderberg (Harvard); M. Lakićević \& J. van Loon (Univ. of Keele); K. Long, J. RomanDuval, \& K. Gordon (STScI); P. Lundqvist (Stockholm Obs.); I. Martí-Vidal (Chalmers Univ. of Tech); J. Marcaide (Univ. de Valencia); C.-Y. Ng (Univ. of Physics, Hong Kong); S. Park (Univ. of Texas, Arlington); M. Otsuka (ASIAA); K. Sandstrom \& S. Hony(MPIA); B. Babler (Univ. of Wisc.); T. Nozawa (Univ. of Tokyo)
} 


\section{Introduction}

When we proposed for the HERschel Inventory of the Agents of Galaxy Evolution (HERITAGE) in the Magellanic Clouds [1], we did not expect to detect SN 1987A. We extrapolated the spectral energy distribution (SED) of the Spitzer measurements of SN1987A [2], and predicted the emission would fall below our sensitivity limit. Thus the submillimeter detection of SN1987A by [3] in the full HERITAGE dataset was a surprising discovery. Indeed, there are two peaks in the SED of SN1987A. The circumstellar dust ring emission peaks at the shorter wavelengths of Spitzer ([4]; [2]). Matsuura et al. interpreted the far-IR/submm SED emission peak to arise from the stellar ejecta of the supernova [3]. They estimated between $0.4-0.7 \mathrm{M}_{\odot}$ of dust mass in the ejecta to account for the far-IR/submm emission, which is the largest dust mass measured for any supernova or supernova remnant.

\section{ALMA and Herschel measurements of the continuum}

Many were skeptical of our argument that the HERITAGE far-infrared/submillimeter emission of SN1987A was thermal emission from freshly formed supernova dust. Some wondered if it were possibly part of the circumstellar emission if it was indeed associated at all with SN 1987A. Thus we pursued ALMA observations of the continuum emission from SN 1987A. The full report on the ALMA continuum measurements for SN 1987A was recently published in [5]. Figure 1, which is from[5], shows the ALMA measurements at $2.8 \mathrm{~mm}, 1.4 \mathrm{~mm}, 870 \mu \mathrm{m}$ and $450 \mu \mathrm{m}$ in comparison with prior measurements at low-frequency radio, optical and X-ray wavelengths. The prominent ring in the optical, X-ray and radio images is the circumstellar material, ejected by the progenitor star, and shocked by the supernova blast wave. The supernova ejecta can be seen at a fainter level inside of this ring of emission.

The ALMA continuum image has two distinct components: the ring (a.k.a. the torus) and the inner ejecta. The ring emission dominates at 2.8 and $1.4 \mathrm{~mm}$ and can be seen at $870 \mu \mathrm{m}$. The inner ejecta emission dominates at 450 and $870 \mu \mathrm{m}$, and if one subtracts the ring emission from the 1.4 mm image, it can be detected as well. The SED of the ALMA bands also reveals these two distinct components (Figure 2). The circumstellar ring appears brightly at the longer wavelengths where the synchrotron emission dominates and this is labeled with green points as the ALMA:torus in Figure 2. When a model for the synchrotron ring emission is subtracted from the ALMA images, the remainder of the flux is determined to be dust emission from the ejecta and is labeled with red points in the SED of Figure 2. Combined with the earlier Herschel continuum data from [3], the $\mathrm{SED}$ of the inner ejecta is fitted for a dust mass of $0.23 \pm 0.05 \mathrm{M} \odot$ plus $0.39 \mathrm{M} \odot$ of amorphous silicate (the silicate mass is not well constrained due to its lower submillimeter emissivity). The amorphous carbon dust emission dominates at the ALMA wavelengths in this model. Thus the ALMA data confirms both the association of the HERITAGE detection with the inner ejecta and the dust mass derived by [3].

Follow-up Herschel observations were also pursued by Matsuura, Barlow and the SN1987A Team. Of particular interest was the $70 \mu \mathrm{m}$ emission which was not detected by the Spitzer SAGE survey and should lie on the Wien edge of the modified black body of the ejecta dust emission. 


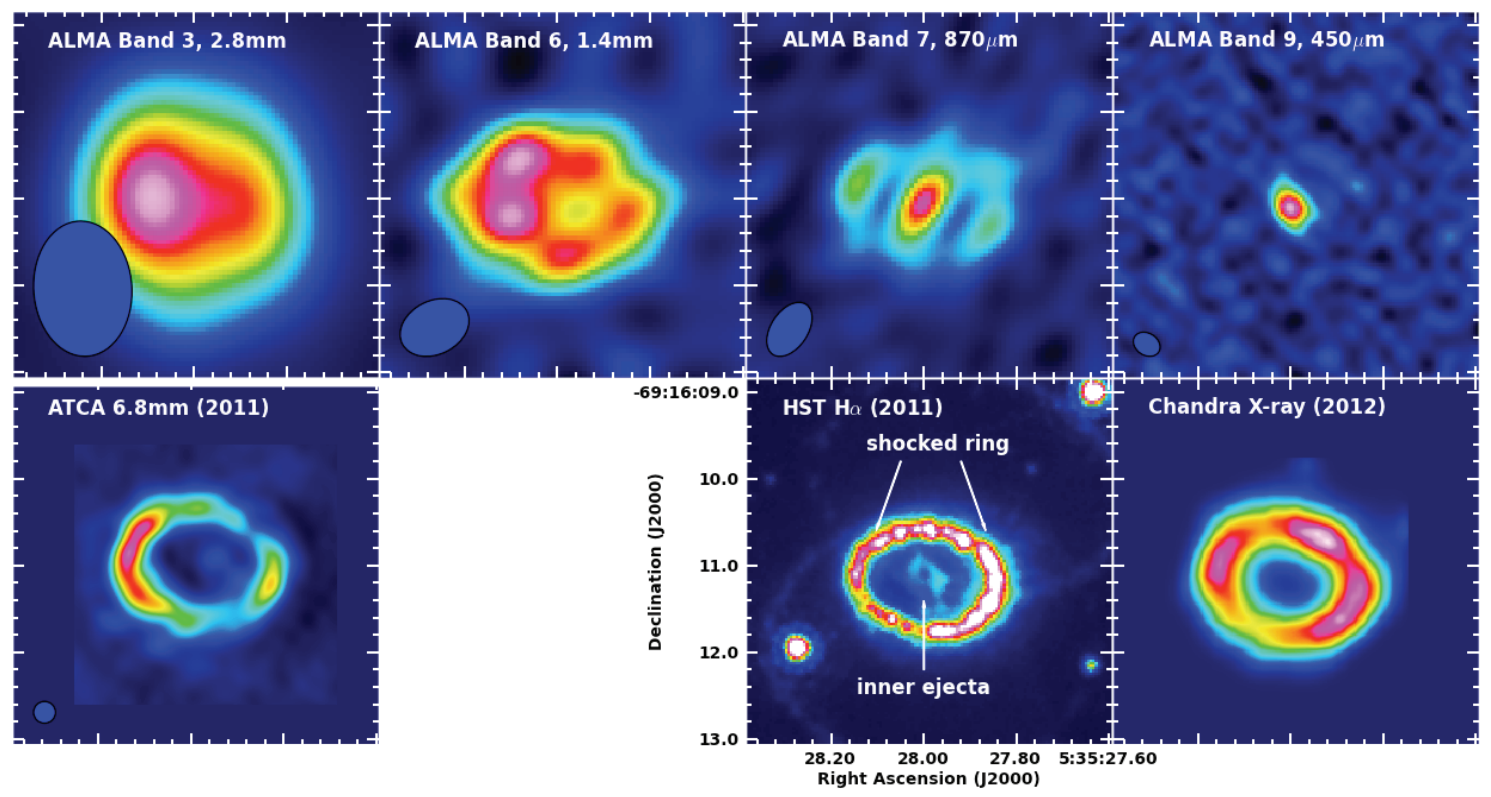

Figure 1: ALMA observations of SN1987A in 4 bands (top) compared to tracers at other wavelengths (bottom). The ring is dominant at the longer wavelengths dominated by synchrotron radiation. The dust emission from the inner ejecta dominates the shortest wavelengths of the ALMA bands [5].

Matsuura et al. report new detections at 70 and $500 \mu \mathrm{m}$ and reveal an SED consistent with their earlier work [6].

\section{ALMA and Herschel measurements of the molecules}

The far-IR and submm range of the initial Herschel detection also covers lines of prominent molecular line transitions. Thus we also pursued follow-up spectral line observations with both ALMA and Herschel to determine how much if any contaminating line emission was present in the bands. Kamenetzsky et al. reported the discovery of the rotationally excited lines of $\mathrm{CO}$ and $\mathrm{SiO}$ in the ALMA data of SN1987A and the follow-up Herschel spectroscopy data of the higher CO rotational transitions (Figure 2, [7]). The line emission does not dominate the continuum. The $\mathrm{CO}$ mass and temperature are estimated at $M_{\mathrm{CO}}>0.01 M_{\odot}$ and $T>14 \mathrm{~K}$. Matsuura et al. also confirms that the $70 \mu \mathrm{m}$ has little line emission from the [OI] $63 \mu \mathrm{m}$ line [6].

\section{Summary}

The follow-up ALMA and Herschel observations confirm the initial Herschel detection of dust emission associated with the ejecta of SN 1987A. The dust mass estimates of 0.3 to $0.7 \mathrm{M}_{\odot}$ for SN 1987A are the highest measured to date for SNe. These high dust masses support the idea that SNe are important dust contributors for high redshift galaxies. The surprising discovery of rotationally excited molecules of $\mathrm{CO}$ and $\mathrm{SiO}$ opens a door to future investigations of the molecular content in SN 1987A. 

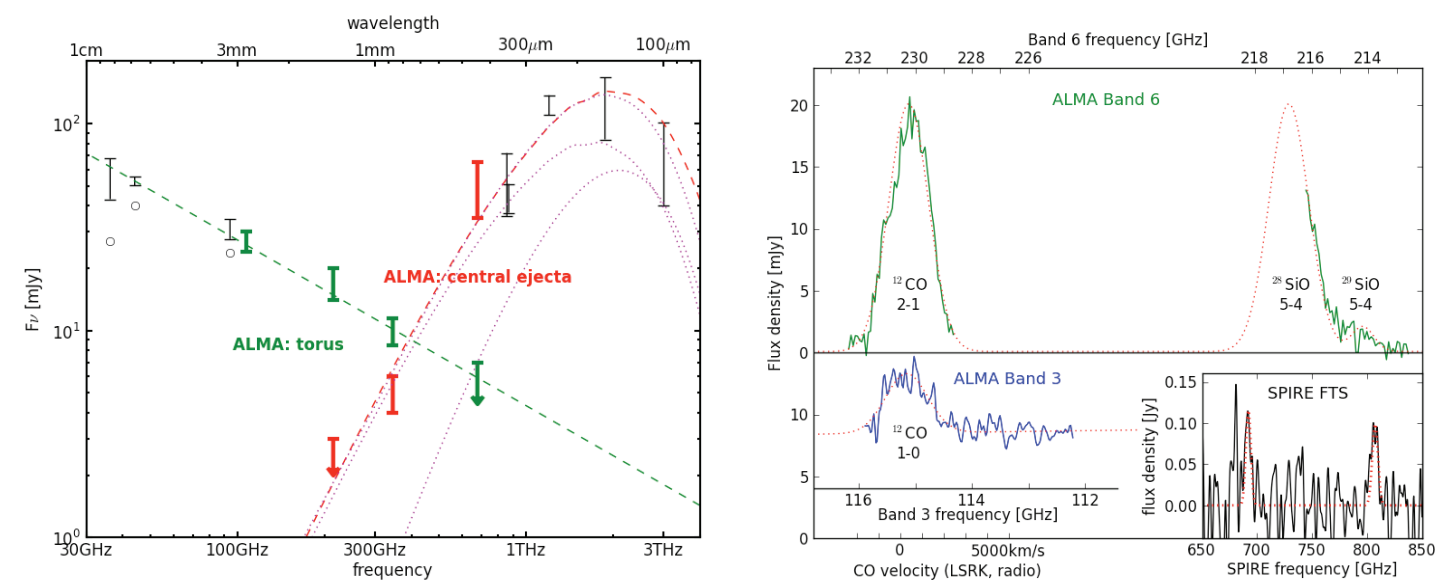

Figure 2: Left: ALMA data in the SED of SN1987A separating the inner ejecta dust emission from the synchrotron emission from the torus (reproduced from Indebetouw et al. 2014). The dashed red line is a model fit of $0.23 \pm 0.05 \mathrm{M} \odot$ amorphous carbon dust at $26 \pm 3 \mathrm{~K}$. The purple dotted lines are a model fit with $0.24 \mathrm{M} \odot$ amorphous carbon dust at $22 \mathrm{~K}$ plus $0.39 \mathrm{M} \odot$ silicate dust at $22 \mathrm{~K}$. These results confirm our dust mass estimates from [3]. Right: The first detection of rotational $\mathrm{CO}$ and $\mathrm{SiO}$ molecular emission in SN1987A (figure reproduced from [7]).

\section{References}

[1] Meixner, M., Panuzzo, P., Roman-Duval, J., Engelbracht, C., Babler, B., Seale, J. et al. 2013, AJ, 146, 62

[2] Dwek, E., Arendt, R.G., Bouchet, P., Burrows, D.N., Challis, P. et al. 2010, ApJ, 722, 425

[3] Matsuura, M., Dwek, E., Meixner, M., Otsuka, M., Babler, B., et al. 2011, Science, 333, 1258

[4] Bouchet, P., Dwek, E., Danziger, J., Arendt, R.G., De Buizer, I.J.M. 2006, ApJ, 650, 212

[5] Indebetouw, R., Matsuura, M., Dwek, E., Zanardo, G., Barlow, M.J. et al. 2014, ApJ, 782, L2

[6] Matsuura, M., Dwek, E., Barlow, M.J., Babler, B., Meixner, M., et al. 2014, ApJL, submitted

[7] Kamenetzky, J., McCray, R., Indebetouw, R., Barlow, M.J., Matsuura, M. et al. 2013, ApJ, 773, L34 